\title{
Assistive Technology Android Application for Autism Spectrum Disorder Therapy
}

\author{
Sumanth Simha C. \\ BE Student \\ Dept. of CSE \\ BMSCE, India
}

\author{
Samarth S. \\ BE Student \\ Dept. of CSE \\ BMSCE, India
}

\author{
Harish Chandra G. R. \\ BE Student \\ Dept. of CSE \\ BMSCE, India
}

\author{
Indiramma M., PhD \\ Professor \\ Dept. of CSE \\ BMSCE, India
}

\begin{abstract}
The power of augmentive and alternative communication through mobile devices like phones, tablets and iPods has changed the lives of children with autism by helping them to cope with their visual, mental and developmental disabilities. Autism Spectrum Disorder in short ASD is a spectrum of closely related disorders with impairment to some degree in social skills, empathy, communication and flexible behavior leading to restricted activities and interests. Extensive research over the years has proven that the use of android and iphone apps with interactive technologies for autism therapy has been quite efficient. This paper provides a supportive tool to counter the motor related disabilities of the ASD children by providing Assistive Technology in the form of an android app. The touch screen interface is very appealing and easy to use due to strong visual support compared to the traditional teaching systems. The app is designed with the learning and evaluation modules by provide all round support to children, parents and tutors.
\end{abstract}

\section{Keywords}

Autism Spectrum Disorder, Assistive Technology, android app, evaluation module.

\section{INTRODUCTION}

Autism Spectrum Disorder popularly called as ASD is characterized by changes in the behavior of the person which is associated with multiple severity stages in early years of life. This disorder occurs with a group of alterations in the age between 7 and 12. ASD is a life-long disability and is divided into three categories: Autistic Disorder also known as Classical autism, Asperger Syndrome and Pervasive Development Disorder-Not otherwise specified (PDD-NOS) or atypical autism[2]. According to the Diagnostic and Statistical Manual of Mental Disorders (DSMIV)[4], the following include the Diagnostic criteria for Autistic Disorder: A. Impairment in social interaction: B. Impairment in communication: C. Restricted and Repetitive behavior: If all these 3 symptoms overlap, then, that child is said to have ASD according to DSM-IV.[5]. The disorders like Sensory Integration Disorder (SID), Dyspraxia, Attention Deficit Hyperactivity Disorder (ADHD) and difficulty in emotion recognition accompany the spectrum of autism disorders. Surveys and publications in the year 2009 showed that 1 child out of every 150 or 110 out of 10,000 were contracted with autism[1].In March 2013 the Center for disease control and prevention showed that 1 in 50 had ASD. March 2014 had 1 in 68 cases diagnosed with Autism Spectrum Disorder [1]. Due to these steep increase in the numbers, many Assistive Technology solutions are being done to make the ASD affected children to be independent when they reach the age of adulthood and perceive others behaviors and feelings and respond to them accordingly. Temple Grand in her book 'Thinking in Pictures and Other
Reports from my Life with Autism' [6] has described how autism children visualize things in the form of pictures. Articulating these facts, we have developed Assistive Technology which essentially is a therapeutic and educational tool for social, communication and motor skill development. It also manifests as a recreational activity for improvement in their daily living conditions. The Assistive Technology app, intends to focus on two finger pinch, tap and touch related games and activities involving pictorial assistance and an evaluation module for the parents and the tutors to track the progress of autistic children. Therefore, the app collectively helps parents, tutors and medical experts effectively involve and participate in tailoring the app to suit their child's autistic needs and help in combating autism.

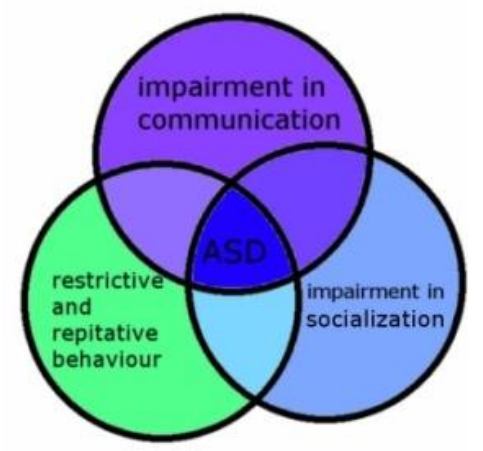

Fig 1: DSM-IV Definition of Autism [4]

\section{RELATED WORK}

There are some existing android and iphone apps for autism but each one of them are deficient in one feature or the other. The TOBY Autism app[7] concentrates on a 2-4 years autism child which targets at improving social, language and cognitive skills through IPod tasks, play activities and rewards. Most of the images in TOBY are virtual images rather than real world images. The Proloquo2Go [8] uses Augmentive and Alternative Communication (AAC) to counteract child's speech problems. This app concentrates on the social skills and not on improving the child's motor ability. Touch Chat is a specifically designed app for children with ASD primarily suffering from Down's syndrome [9] and for toddlers. This app is disorder specific and does not focus on children of all ages. Alexicom AAC for Android lets you use Android phone or tablet as augmentive communication device. It has over 1,200 pre-made pages and 7,000 images in the public libraries.

The major shortcomings of the above listed apps are:

1. Designed only for a particular disorder but are found ineffective in treating a wide range of autism spectrum disorders. 
2. There is no provision for two finger pinching action which is found to be effective in coordination of fingers.

3. The above apps just display some images and make the child to answer some questions on those. The problem is that the child is not able to generalize things. For e.g.: Given the image of a bike, the child registers in its mind that that's the only kind of bike available. Given another kind of bike, the child is not able to recognize it as ASD children suffer from generalization problem.

3. There is minimal parent-child or tutor-child interaction as they cannot include real world images or the images found in and around them in the app for better generalization and object recognition.

4. Minimal options to track the progress of the child on activity basis or on a weekly basis.

\section{PROPOSED WORK}

\subsection{Overview}

The Assistive Technology app for autism is designed such that each level caters to some particular disorders in the set of autism spectrum disorders. The app framework can be viewed as shown in the figure 2. The entire app is divided into four levels. Level 1(Learn Activity) has a variety of images for visualization of nearby and real world objects with animation and motivating sounds which treats the speech and communication impairment through generalization of objects and Attention Deficit Hyperactivity Disorder(ADHD)[11] by catching their attention for a long time. It involves the use of finger movements like swipe and tap. Level 2(Match Activity) deals with Sensory Integration like hand-eye coordination, finger muscle coordination and Dyspraxia- a neurological disorder [10] by implementing touch and two finger pinching action. Level 3(Outline Activity) deals with object recognition; outline matching with two finger pinching technique which assists them to fight Dyskinesia and other finger and motor coordination related disorders. Level 4(Coloring Activity) helps the child to increase its motor skills through touch and tap and better color and object recognition. The ASD Therapy app is designed kept in mind the autistic children of all ages and all neurological disorders. The parents and tutors can view the child's progress on a weekly basis in the evaluation module, recognize the child's area of difficulty, the level of disorder and provide appropriate treatment.

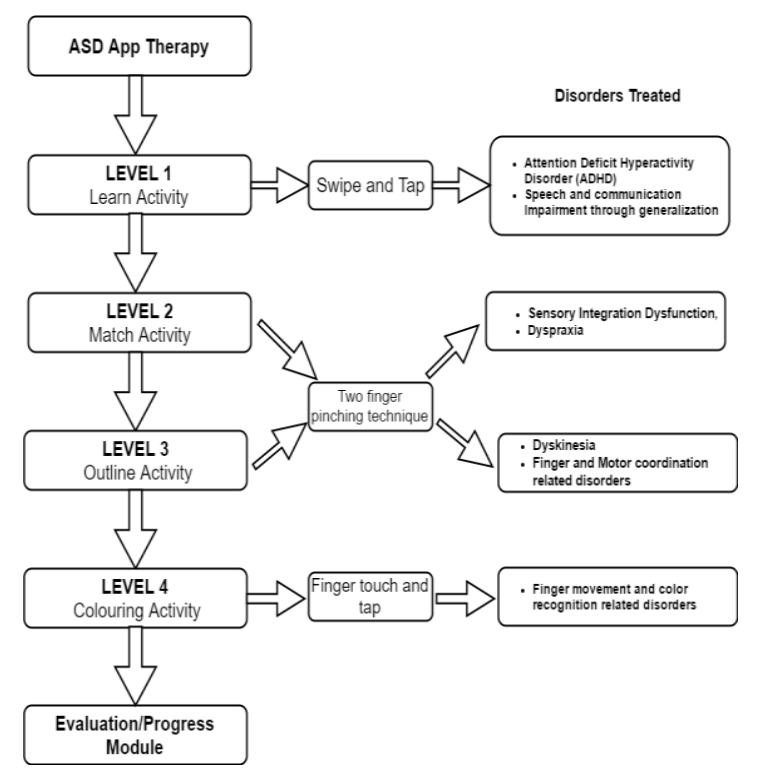

Fig 2: Assistive Technology App Flow Diagram

\subsection{Working of the Assistive Technology Application}

The main screen of the app shows four levels LEVEL 1, LEVEL 2, LEVEL 3 and LEVEL 4 with some 2D animation and appealing background sounds and image as in figure 3. The app has been broadly classified into 4 levels depending upon the age and the area where the autistic child is facing the difficulty.

LEVEL 1 (Learn Activity): Level 1 is basically designed for autistic children of ages 5 to 10 years. The ASD children have problem in generalization and visualization of day to day objects. For e.g.: given a particular pen , the child recognizes it but when given another pen the child fails because it not able to generalize objects. To solve this problem, a comprehensive set of assorted images are provided with their names as labels and with Text to Speech implementation which spells out the name of the object in English, US. When the user clicks on Level 1 on the main screen, the activity with different categories of objects for the child to learn is opened. The important categories are alphabets, animals, shapes, fruits, vehicles, houses, stationary, emotions, etc.
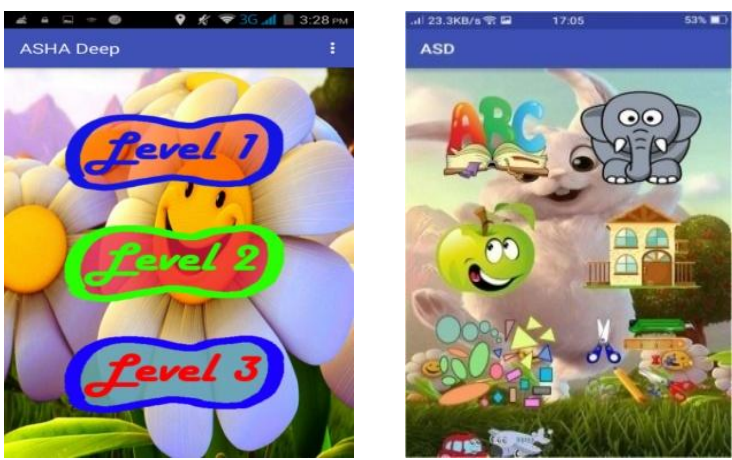

Fig 3: Main Screens of the App

When the thumbnail is clicked, all the categories of images under the main category are displayed. For e.g., if the fruits thumbnail is clicked all types of fruits are displayed and so on. When the apple image is clicked, different images of apple one by one is displayed with the name of the object spelt out as shown in figure 4 . Real life images are added so that the child will be able to generalize real world objects. The child will be able to view the same images that it would have seen in real life and will be able to relate well. Attention Deficit Hyperactivity Disorder is also taken care the images are attractive with motivational sounds and will catch the child's attention for a long time. The same images will be used by the parent as well as the tutor in teaching. Hence, there will be less confusion for the child in recognizing the images. Figure 4 clearly explains the implementation of the Learn Activity.

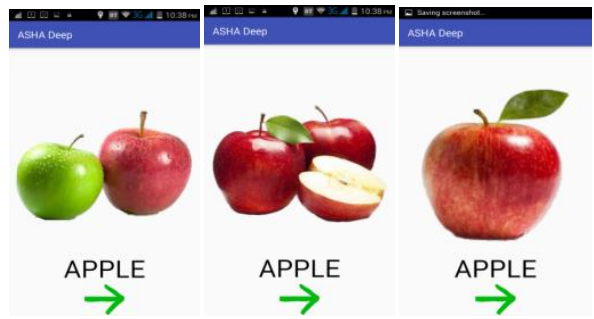

Fig 4: Learn Activity 
LEVEL 2 (Match Activity): Level 2 basically is targeted at the children of age 10-12 years. Dyspraxia, a term used to state the inability to initiate, organize and perform movements like thumb and index finger movement, holding pencil or pinching action. The autistic children suffer with Sensory Integration Dysfunction (SID) like hand-eye, muscle and motor coordination. These disorders are looked at by level 2 of the ASD app. Now, when the user selects animals, the screen displays a main image at the top of the screen, and a set of 3 images at the bottom. The user has to pinch one of the 3 images by using two finger pinching technique that matches with the main image. If the user pinches on the wrong image, a red colored cross symbol is displayed on the image and the phone vibrates giving an indication of the wrongly selected image. If the user pinches the right image, the app moves to another screen, in which a smiley appears with claps which motivates the user to continue as in figure 5. Also, to keep track of the users performance, the app provides a star for every right question. This happens for every 5 questions the user answers, which makes the app act like a remedy for the disorders mentioned above and just not like a timepass game. The two finger pinching technique proves to be quite effective in hand eye coordination and finger coordination related disorders.

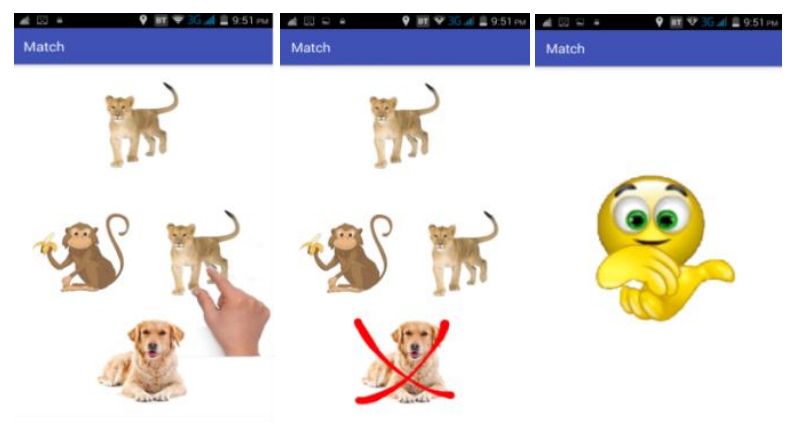

Fig 5: Match Activity

LEVEL 3(Outline Activity): This level of the app deals with higher level of training the ASD children who are comfortable with the learn and match activities. Object Recognition, Dyskinesia- difficulty or distortion in performing voluntary movements and Sensory Processing Disorders are dealt in this level. It basically involves the user to match the image with its outline. The matching is done by the user by pinching the right image using two fingers that fits into the outline. This level is developed to train the ASD children in an advanced manner remedying for all the disorders mentioned in levels 1 and 2 . For illustration purpose, let's assume that the user has selected animals. This leads to the screen where the exercise comprises with a set of images below an outline. The user has to pinch the right image that fits the outline. If the user pinches on the wrong image, a red colored cross symbol is displayed on the image and the phone vibrates giving an indication. If the user pinches the right image, the image moves and fits into the outline as in figure 6. The app provides a star for every right question. This happens for every 5 questions the user answers. This enables the child to relate the image with its corresponding silhouette.

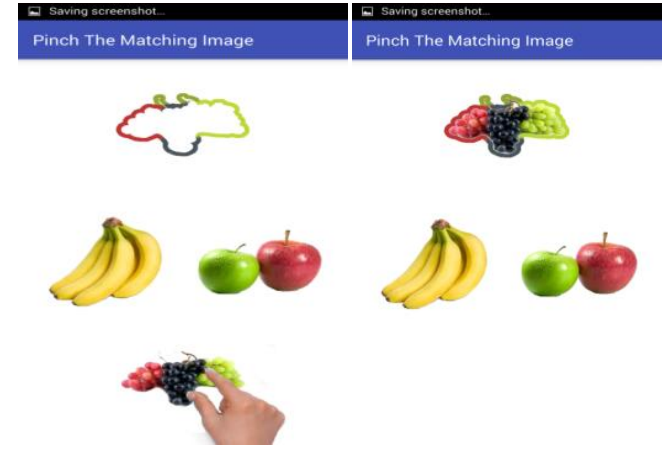

Fig 6: Outline Activity

LEVEL 4(Coloring Activity): This level is mainly designed for autistic children with finger related disorders and color recognition. Repetitive finger movements, hand flapping and flicking fingers are some of the finger related disorders. Autistic children are also weak in hand movement due to delayed sensory perception. This activity is designed taking all these disorders into account. The level displays the outline of an object as shown in figure 7 . The child has color the outline by one finger touch and tap mechanism and care is taken such that the shading does not exceed the outline. After $90 \%$ of the outline is full, the object is displayed and the next outline appears. Through this activity, the child would be able to overcome its touch aversion and would be able to recognize colors and objects. The parents and tutors can decide different outlines from different categories with varying difficulty based on the child's response. They can slowly increase the difficulty level as the child progresses.
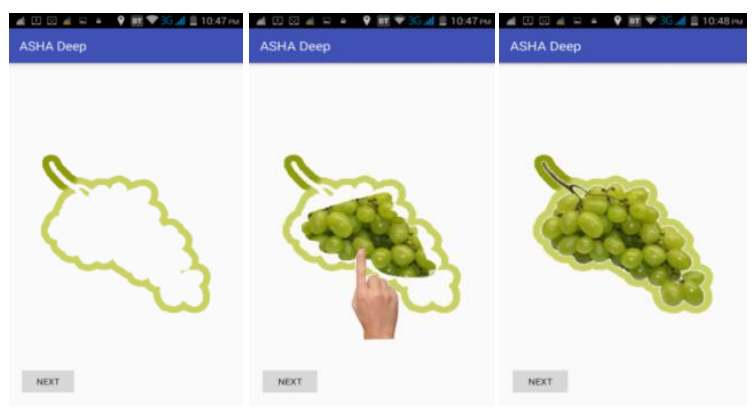

Fig 7: Coloring Activity

\subsection{Provision to capture real world images}

The main screen of the app allows the tutor or the parent to select the camera to capture the image, crop it according to their needs and make a new category or add them into the existing sub categories if they find that the existing images are insufficient as in figure 8 . The newly added images come along with the existing categories when the app is opened. Therefore, along with the images already provided, the tutors can tailor the app based on their needs as well as by taking into account the level of disorder of the child. This is mainly useful while dealing with emotions. The app enables the parent/tutor to capture photos and attach emotion data with the captured photo. When the images of the child or its family are taken and placed under the emotions category, the child would be interested to learn and recognize the emotions well. 

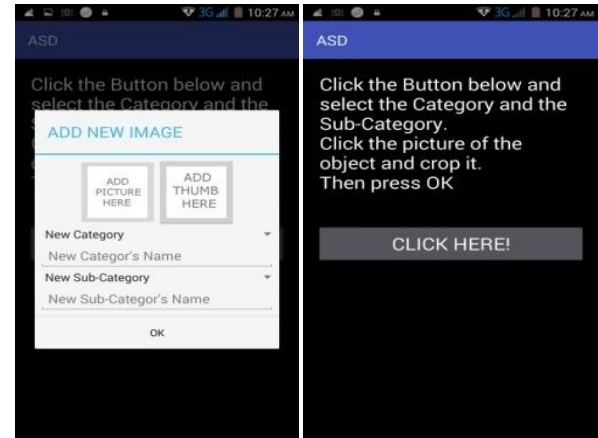

Fig 8: Provision to capture real world images

\section{TECHNICAL DETAILS OF THE APP}

Google's Android Studio was used to build the app. The code was written entirely in Java and layout resources were written in XML. For pinch support, a custom view was created which supports the pinch gesture. Every image that can be selected by a pinch gesture is an instance of the above said view. This view handles the touch events sent to it on its own. A pinch gesture is considered to have started when two fingers have touched the screen. When the distance between the fingers reduces by a fixed number of pixels then the pinch is considered to be a success. Since the app had a huge requirement of good quality images we have tailored the app in a way so that the images are not packaged into the apk. The images are downloaded from an external location on running the app for the first time. The major challenges were in designing the Layout since android devices vary in screen size and dimensions and our app needed proper dimensions for each element we could not have a single layout resource which could be displayed on screens of all sizes and dimensions. This was overcome by hard coding the distances between the margins and each of the views and also their dimensions. That is all the measurements are done during the run time and then used to display the elements on the screen.

\section{RESULTS}

The (Peabody Developmental Motor Scale) PDMS-2 scale consists of different subsets for assessing the gross and fine motor skills of different ages of children from their early childhood. The Gross motor skills include the abilities of large muscle systems to move from place to place which includes the movement and balance skills like the ability to crawl, walk and run. Fine motor skills include skills like grasping an object, motor coordination and visual motor integration. The Assistive Technology app focuses on the improving the fine motor skills compared to the gross motor skills. The subsets used to evaluate the child's fine motor skills are derived from the PDMS-2 scale their and implementation is listed below:

Attention time: The ASD contracted children have ADHD (Attention Deficit Hyperactivity Disorder) where they have a very short attention span, are restless and they are easily distracted. The attention time is evaluated from the Learn Activity by logging the amount of time their eyes are oriented towards the screen. Once they take off their eyes from the device, the time recording stops.

Motor Delay: Motor Delay is calculated from the Match and Outline Matching activities by using the two finger pinching technique. The time difference between the child placing the thumb and the index finger on the screen and the time required to those fingers together to perform the pinching action is taken as the motor delay.
Object Recognition: The ability of the child to recognize the objects that it has learnt in the Learn Activity is assessed in the Match Activity by taking the time and scores into consideration.

Grasping Time: The ability of the child to remember the objects shown in the Learn Activity and its ability to generalize the objects that it has learnt and apply it to any real world situation is taken to measure the grasping power.

The experiment was conducted on a group of ASD contracted children belonging to the ASHA (Academy for Severe Handicaps and Autism) foundation each belonging to an age group of 4-6 years. The app was tested on children with varying levels of ASD varying from verbal functioning child to a non verbal child. Every child was made to sit on a chair and the tablet consisting of the Assistive Technology app was placed on the table to provide proper body equilibrium for the child to operate the app. Each child was made to play each of the following activities in the app and the scores for the subsets listed above were captured by the app. Based on the data collected, computations were performed at the server side and the child's progress was evaluated based on different subsets. An example of the graph plotted by the app for one child under the subsets motor delay, grasping time, object recognition time and attention time is shown in figures 9,10 , 11 and 12.

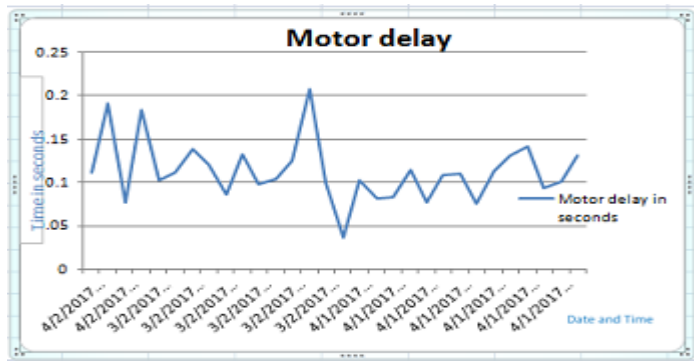

Fig 9: Graph of time in seconds versus Date and Time plotted for Motor Delay

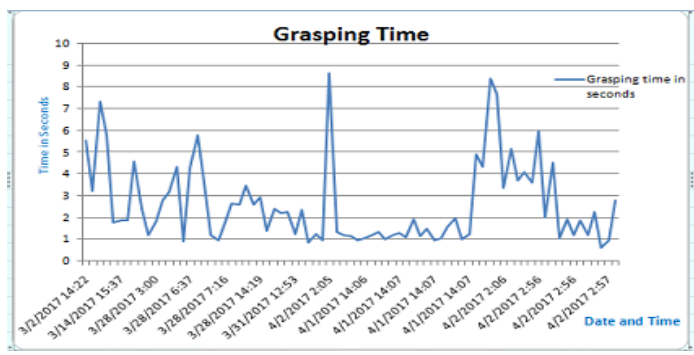

Fig 10: Graph of time in seconds versus Date and Time plotted for Grasping Time

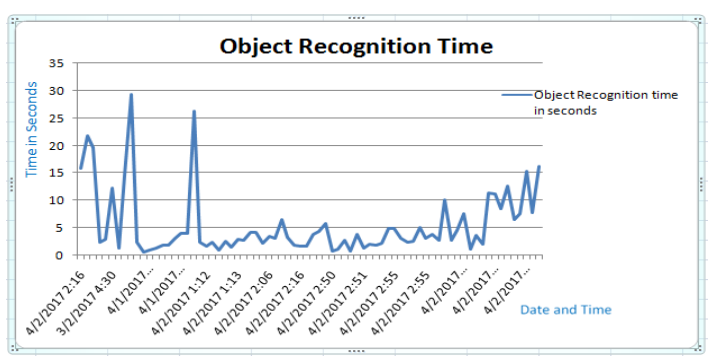

Fig 11: Graph of time in seconds versus Date and Time plotted for Object Recognition Time 


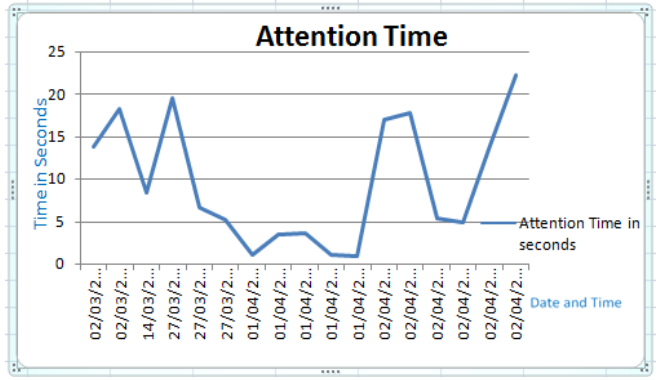

Fig 12: Graph of time in seconds versus Date and Time plotted for Attention Time

\section{CONCLUSION}

This paper aims at developing a fully packaged mobile application for Autism Spectrum Disorder (ASD) Children suffering from persuasive neurodevelopment disorders. This paper proposes different levels which are categorized based on the age as well as the varying level of disorder in the ASD counteracted children and also provides support for parents and tutors by generating weekly reports of the performance of the children through the learning and evaluation modules.

The application can be further enhanced by collecting the brain signals using the EEG Machine and then analyzing the data using Machine Learning techniques. Hence, through such apps and with the continual support of therapists innovative solutions can be provided to autism. There is a need for creating awareness in the society about such apps so that the parents and tutors can use these apps in their homes and in their schools to treat their autistic children.

\section{ACKNOWLEDGEMENT}

We would like to greatly acknowledge the support extended by teachers, coordinators and ASD Children during our interaction and demo of the Assistive Technology app at ASHA (Academy for Severe Handicaps. and Autism) charitable trust providing special education needs to children with autism in Bangalore.

\section{REFERENCES}

[1] Technologies as Support tools for Persons with Autistic Spectrum Disorder: A Systematic Review - Nuria Arestin Bartolome and Begonaya Garcia Zapirain.

[2] Interactive Mobile Technology for Children with Autism Spectrum Condition (ASC)

[3] Young Children with ASD- Amy Mathews, PhD and Jamie Omen De Schryver, PhD, Grand Valley State University

[4] South West Autism Network www.swanautism.org

[5] The Autistic Spectrum: Characteristics, Causes and Practical Issues

[6] 'Thinking in Pictures and Other Reports from my Life with Autism' - Temple Grandin

[7] TOBY Autism Therapy www.tobyplaypad.com

[8] Proloquo2Go www.assistiveware.com

[9] Down's Syndrome and Language Development- Colleen D. Oliver Southern Illinois University Carbondale, oliverc@siu.edu

[10] Dyspraxia or developmental coordination disorder? Unraveling the enigma - John Gibbs, Jeanette Appleton, and Richard Appleton

[11] The Co-Occurrence of Autism and Attention Deficit Hyperactivity Disorder in Children - What Do We Know?-Yael Leitner. 